\title{
Abnormal segregations in crosses between two cultivated rice species
}

\author{
JEAN-LOUIS PHAM* \& BRUNO BOUGEROL \\ Laboratoire d'Amélioration des Plantes, Centre ORSTOM d'Adiopodoumé, BP V51 Abidjan, Côte d'voire
}

\begin{abstract}
An $\mathrm{F}_{1}$ hybrid between the two cultivated rice species Oryza glaberrima and $O$. sativa spp. japonica was pollinated by four different $O$. sativa genotypes to obtain four back-cross progenies with 9-36 plants each. These progenies were followed for genetic marker segregations. Segregation distortions were demonstrated at several loci. Preferential allele associations were detected. Some distortions could be explained by the sterility of the female gametes of the $F_{1}$ hybrid caused by a sporogametophytic interaction gene, while an early differential zygotic selection could be the origin of the other distortions.
\end{abstract}

Keywords: genetic resources, interspecific hybrids, Oryza glaberrima, Oryza sativa, reproductive barriers, segregation distortion.

\section{Introduction}

Rice offers a rare opportunity to study the relationships between two cultivated species belonging to the same genomic group (AA genome, Morinaga, 1964). Furthermore, since rice is a major crop, this is not only of theoretical interest but also important for the use of rice germplasm.

The analysis of isozyme polymorphism (Second, 1982) and those of chloroplastic RFLPs (Dally \& Second, 1990) and nuclear RFLPs (Wang et al., 1992) support the hypothesis of distinct domestication of the two cultivated rice species, in Asia for Oryza sativa and in West Africa for Oryza glaberrima (for review see Oka, 1988). Agronomical studies revealed useful characteristics in some $O$. glaberrima cultivars, such as early tillering (observations of plant breeders), tolerance to drought (Sano et al., 1984), tolerance to Rice Yellow Mottle Virus (Attere \& Fatokun, 1983; John et al., 1985; John \& Thottapilly, 1987) or some insects (Vercambre, 1982; Sauphanor, 1985). However, despite these characteristics, it is the distinct genetic origin of $O$. glaberrima that has been used to argue that the African species is a potential source of improvement for $O$. sativa. This idea is confirmed by the results of

Correspondence: Jean-Louis Pham.

*Present address: Jean-Louis Pham, Station de génétique végétale, INRA-UPS, La Ferme du Moulon, 91190 Gif-sur-Yvette, France.
Silue \& Notteghem (1991) who tested $99 O$. glaberrima accessions for blast resistance and who observed for some accessions a resistance that was not higher than that of $O$. sativa but different.

The use of $O$. glaberrima for improvement of $O$. sativa is difficult because of strong reproductive barriers between the two cultivated species (Chu et al., 1969; Sano, 1986; Yabuno, 1990). Sano et al. (1980) showed a tendency to the non-independent transmission of morphological and viability traits in the progenies of crosses between $O$. glaberrima and $O$. sativa. However, the influence of reproductive barriers on the variability of progenies obtained from such crosses remains poorly known.

In a previous paper (Bougerol \& Pham, 1989), we studied the influence of the O. sativa genotypes on the characteristics of $\mathrm{F}_{1}$ hybrids between $O$. glaberrima and $O$. sativa. This paper reports a part of a study of the influence of the $O$. sativa parent on back-cross progenies. We used an $\mathrm{F}_{1}$ hybrid, O. glaberrima $\times O$. sativa spp. japonica, as constant female parent and studied the back-cross progenies obtained after pollinating with four different $O$. sativa genotypes. We present here our results dealing with the segregations of genetic markers observed in the four progenies. Although the number of plants studied is low (from 9 to 36 depending on the progeny), it is, as far as we know, the first time that so many plants have been studied from back-crosses involving O. glaberrima and O. sativa. 


\section{Materials and methods}

\section{Plant material, cultivation and experimental design}

Four $O$. sativa genotypes were used and were classed into the indica and japonica subspecies based on isozyme studies (Second, 1982; Ghesquière \& Second, 1983; de Kochko, 1987). These are ES70-6 (Tanzania, japonica), YS309 (Guinea, japonica showing presumed introgression from $O$. longistaminata), YS45-1 (Guinea, japonica) and SS404 (Senegal, indica). These traditional African cultivars were obtained from the ORSTOM $\dagger$ collection. The $O$. glaberrima genotype WO25 was provided several years ago by the National Institute of Genetics (Misima, Japan) and was used to study the relationships between $O$. sativa and $O$. glaberrima (Sano et al:, 1979; Sano, 1983). The purity of parental lines was ensured by successive generations of controlled self-fertilization.

Initially, the parents WO25 (female) and ES70-6 (male) were crossed to obtain $F_{1}$ hybrids. No difficulty was observed at this step. The $F_{1}$ plants showed a very low pollen fertility ( $<1$ per cent) which is usual in such crosses (Morishima et al., 1962; Chu et al., 1969; Yabuno, 1977; Bougerol \& Pham, 1989) and a complete seed sterility was observed on the panicles that were not used in crossing. These $F_{1}$ hybrids were probably female-sterile (Chu et al., 1969) since it was difficult to obtain seeds by pollinating the $F_{1} s$ with each of the four $O$. sativa parents. The rate of successful back-cross pollination was about 4 per cent and did not differ significantly between back-crosses. This rate is similar to that observed by Sano (1983). Twelve to 46 seeds per progeny were obtained. As the female parent was constant, each of the four progeny will therefore be denoted according to the male parent: //ES70-6, //YS309, //SS404 and //YS45-1.

Seeds were dehulled, disinfected in a solution of sodium hypochlorite, rinsed in distilled water, and sown in Petri dishes with nutrient solution. After 10 days, seedlings were planted out in 2-1 pots. Plants were cultivated in a glasshouse with constant irrigation. A randomized block design was used which included the four back-cross progenies, the $F_{1}$ hybrid WO25 $\times$ ES70-6, and parental lines.

\section{Genetic markers}

Six isozyme marker loci and one morphological marker locus were used. The electrophoresis methods on starch gel that were used are described by Trouslot \& Second (1980) and de Kochko (1987). Four enzymatic systems were used: esterases (Est), phospo-

$\dagger$ L'Institut français de recherche scientifique pour le développement en coopération, Paris, France. glucose isomerase $(\mathrm{Pgi})$, catalase ( $\mathrm{Cat})$ and shikimate dehydrogenase $(S d h)$. The Pgi-1 locus is located on chromosome 3, the loci Est-2, Cat-1 and $S d h-1$ are on chromosome 6, and the loci Est-1 and Est-9 are on chromosome 7 (using the unified chromosome nomenclature published in Rice Genetics Newsletter 1990).

The $O$. glaberrima parent WO25 and the $O$. sativa parent YS309 showed spikelets with a red coloured apiculus (locus $C$ ). Following Sano et al. (1979), these genotypes were considered as having the $C$ allele, the other parents having the $C^{+}$allele (non coloured apiculus). The $C$ locus is located on chromosome 6 and is linked to the sporogametophytic interaction locus $S-1$ (Sano, 1986). It is also linked to the isozyme locus Est-2 (about 9 per cent recombination calculated from intra- $O$. sativa crosses, genetic map published in Rice Genetics Newsletter 1987).

\section{Statistical methods}

The conformity of mono-locus segregations to Mendelian proportions was tested following Sokal \& Rohlf (1981) using the binomial test for progenies //ES70-6, //YS45-1 and //YS309 (frequencies <25), and the Chi-square test with continuity correction for progeny //SS404 (frequencies between 25 and 200). The co-segregation of all the pairs of loci were tested (Chi-square test) in the progeny //SS404 only because of the small sample sizes of the other progenies.

\section{Results}

\section{Development of plants}

Plant losses were observed during the cultivation, varying from 37 to 60 per cent depending on the progeny. All the progenies showed a similar proportion of nongerminated seeds $(<20$ per cent). This rate is similar to that observed for the $F_{1}$ hybrid WO25/ES70-6 and to the results of Chu et al. (1969). The second level of loss was at the seedling stage, during the 2 weeks following germination. Significant differences appeared at this stage between the progenies. The highest level of mortality was scored in the progeny //ES70-6 (53 per cent of germinated seeds). It was 29, 19 and 10 per cent in the progeny //YS245-1, //SS404 and //YS309, respectively.

\section{Segregations of genetic markers}

Table 1 shows the results of mono-locus segregations. Out of the 23 observed segregations, 16 agreed with Mendelian proportion 1:1, while 7 were significantly distorted at the 5 per cent level. At least one abnormal segregation was observed in each of the four progenies. 
Table 1 Segregations observed at marker loci for each of the four backcross progenies, and test of their conformity to Mendelian proportions $1: 1$

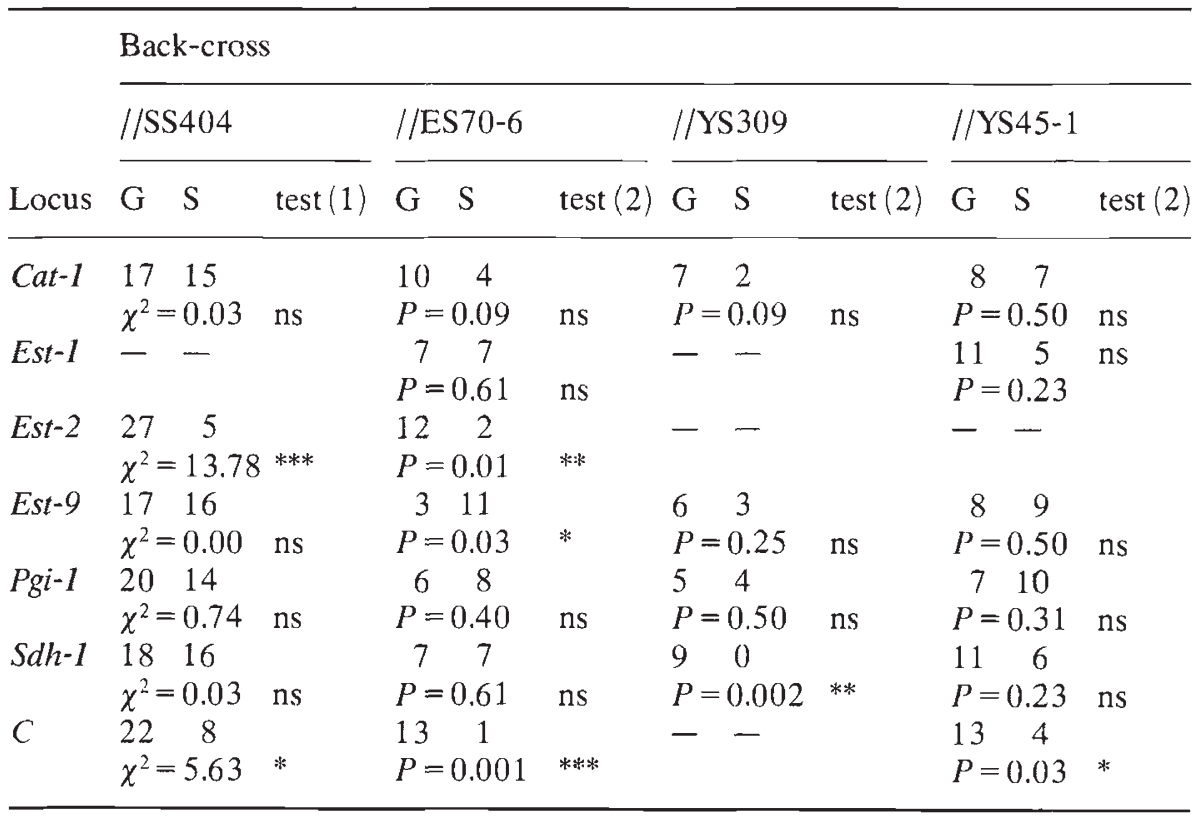

(1) $\chi^{2}$ test with continuity correction.

(2) Binomial test.

G: plants with the allele donated by the $O$. glaberrima parent of the $\mathrm{F}_{1}$ hybrid.

$S$ : plants with the allele donated by the $O$. sativa parent of the $\mathrm{F}_{1}$ hybrid.

ns, non-significant; ${ }^{*}, P<0.05,{ }^{* *}, P<0.01,{ }^{* * *}, P<0.001$.

The linked loci Est-2 and $C$ were subject to distortions in all of the progenies where they were scored: //SS404 and //ES70-6 for the locus Est-2; //SS404, //ES70-6 and //YS45-1 for the locus $C$. An abnormal segregation was observed for $S d h-1$ in the progeny //YS309. All these distortions showed an excess of the allele contributed by the $O$. glaberrima parent WO25. However, an excess of the allele donated by the $O$. sativa parent ES70-6 was observed for Est-9 in the progeny //ES70-6. Thus, this progeny showed two opposite deviations.

Although not significant at the 5 per cent level, trends in deviation from Mendelian proportions were observed for Cat-I in the progenies //YS309 and //ES70-6, for Est-1 and Sdh-I in the progeny //YS451. If one pooled the segregation for Cat-1 of the progenies //YS309 and //ES70-6, a significant excess of the allele donated by WO25 was noted.

All the co-segregations of the pairs of loci studied in the progeny //SS404 were consistent with previous data on the independence of the involved loci (Pham et al. 1990). However, a multilocus analysis showed a tendency towards preferential association of some alleles. In this analysis five independent isozyme loci were considered. There are $2^{5}=32$ possible combina- tions for all the alleles. Only 19 of them were observed among the 29 observed plants. Moreover, if we only considered the four loci Est-2, Est-9, Pgi-1 and Cat-1, seven plants out of 29 showed the same allele combination as the O. glaberrima parent WO25. This proportion differed significantly from the theoretical proportion $1: 16\left(X^{2}=4.994\right.$, 1. d.f., $\left.P=0.025\right)$. However, it did not differ from the theoretical proportion calculated using the hypothesis of a random association of gametes and observed allele progenies.

\section{Discussion}

\section{Analysis of segregation distortions}

Wendel et al. (1987) give several examples of plant species where abnormal segregations were observed. In rice, distortions frequently occurred in crosses within $O$. sativa (Pham et al., 1990) and in crosses between $O$. sativa and $O$. longistaminata (Causse \& Ghesquière, 1991). The skewed segregations of marker loci are caused by the selection against chromosome segments containing these markers. This selection may act either at the haploid gametophyte stages during the prezygotic phase, or after the fertilization. Ottaviano \& 
Mulcahy (1986) defined the gametophytic selection, including fertilization events, and sporophytic selection.

Using isogenic lines Sano et al. (1979) proposed a one-locus sporogametophytic interaction model that explains part of the sterility of the hybrids between $O$. sativa and $O$. glaberrima. The model is the following: the genotypes of 108 (O. sativa spp. indica) and WO25 (O. glaberrima, also used here) are supposed to be $S-1^{a}$ $S-1^{a}$ and $S-1 S-1$, respectively. The genotype of $F_{1}$ hybrid is $S-1^{a} S-1$. The presence of the $S-1$ allele in the maternal tissue leads to the sterility of male and female gametes with the $S-1^{a}$ allele. These authors located this gene ('gamete killer') on chromosome 6 by linkage with the $C$ locus. Sano (1983) then identified the gene $S-3$ on chromosome 11, acting in the same way, but only on the male gametophytes ('pollen killer').

This model of gametic selection can explain the deviations observations for the Est-2 and $C$ loci. Let us suppose that WO25 and ES70-6 have alleles $S-i$ and $S$ $i^{a}$, respectively, acting in the same way as $S-1$ and $S$ $1^{a}$. The excess of $O$. glaberrima alleles at the Est 2 and $C$ loci in the progenies could be explained by a linkage between these loci and the gene $S-i$. Although we cannot demonstrate it, it is likely that $S-i$ is the $S-1$ gene studied by Sano et al. (1979) since the same $O$. glaberrima genotype WO25 was used, and since both are located on chromosome 6.

The observation of Mendelian and non-Mendelian segregations at Est-9 and $S d h-1$ loci depending on the considered progeny demonstrates that the sporogametophytic interaction model is not sufficient to explain the distortions observed in this case. Using this model, the same deviation would be expected in all of the backcross progenies obtained from the $F_{1}$ hybrid, except if one imagines particular correction mechanisms.

It is therefore necessary to consider the genotype of the pollinating parent of the back-cross, i.e. ES70-6 for Est-9, YS309 for $S d h-1$. It is difficult, in the case of gametophytic selection, to imagine a preferential selection of some ovules by the pollen. Since there is only one ovule per spikelet, it would suppose a non independent fertilization of the spikelets. The observation of hybrids between ES70-6 and all O. sativa parents argues against the hypothesis of incompatibility systems.

We may propose the hypothesis of sporophytic selection during the early development of the seeds, at the germination stage or just after this stage. These two last possibilities can be rejected because: (i) the losses observed at this stage are not high enough to explain high segregation deviations and, moreover, these losses are homogeneous between progenies; and (ii) it is unlikely that the mortality of seedlings is the source of distortions at locus $S d h-1$ for the progenies //SS404, //YS45-1 and //YS309. Whereas this mortality rate is roughly the same in these three progenies, they differ for segregations at locus $S d h-1$. Moreover, the mortality rate in the progeny //YS309 cannot explain the magnitude of distortion at this locus. On the other hand, the progeny //ES70-6 shows an high mortality rate, about 50 per cent, that could explain the distortion noted at locus Est -9 . Some examples of weakness or non viability were recorded in the Sativa group after intra- or interspecific hybridization (Chu et al., 1969), but the genetic models proposed cannot be applied here.

These data suggest that the early abortion of the zygote is the more likely hypothesis to explain the distortion at locus $S d h-1$, and perhaps at Est-9. The hypothesis of early differential zygotic abortion was chosen by Rick (1963) and Gadish \& Zamir (1987) to explain abnormal segregations in crosses between Lycopersicon esculentum and Lycopersicon chilense and between Lycopersicon esculentum and Lycopersicon pennellii, respectively. An unfavourable interaction between the endosperm and embryo was suggested to explain such phenomena (Grant, 1975). At the moment, we do not know the genetic basis of the observed differential zygotic selection. A possible way to study this would be the model developed by Chu \& Oka (1970) and Ghesquière (1988) to explain the failure of endosperm development in $F_{1}$ hybrids of crosses between $O$. sativa and the wild species $O$. longistaminata. This model is based on an unfavourable allelic dosage of two genes in the endosperm.

\section{Consequences of abnormal segregations}

The general existence of sporogametophytic interaction genes creates problems for the integration of $O$. glaberrima genes into $O$. sativa. Let us assume that we want to introduce an $O$. glaberrima trait into an $O$. sativa genotype, and that the genes governing this trait are independent of the sterility gene. The production of near-isogenic lines of $O$. sativa will be slowed down by this sterility gene, since the proportion of offspring having an undesirable allele $G$ linked to the sterility gene is equal to $1-p$, where $p$ is the recombination rate between the locus $\mathrm{G}$ and the sterility gene.

The model of sporogametophytic interaction involved in the reproductive barriers between the two cultivated species was also used to explain hybrid sterilities in crosses between the $O$. sativa subspecies indica and japonica (Oka, 1988; Ikehashi \& Araki, 1986). Varieties overcoming the sterility system, called Wide Compatibility Varieties, were found in $O$. sativa 
(Ikehashi et al., 1991). This property of wide compatibility is caused by a 'neutral' allele $S n$. The question is whether it could exist in O. sativa or O. glaberrima varieties compatible with the other cultivated species. As far as we know, no such variety has been detected. Sano (1990) demonstrated the existence of a modifier gene, transforming the action of a pollen killer gene into that of a gamete killer. This mechanism suggests that it could be of interest to study strategies to inactive the pollen killer action.

Even when sterility genes are not involved, restrictions to recombination occur in interspecific crosses (Paterson et al., 1990). The multilocus analysis of the progeny //SS404 clearly showed the excess of some allelic combinations of the WO25 parent. We observed that in all the progenies most of the distortions are in favour of alleles contributed by $O$. glaberrima. Further analyses will be made to study if the same tendency is observed at the morphological level.

Finally, this study shows that interspecific crosses can reveal novel genetic diversity of $O$. sativa, since different levels of zygotic selection were observed according to the $O$. sativa genotype. This underlines the idea that the evaluation of germplasm must include a study of crosssing barriers, in addition to surveys of morphological, biochemical and molecular polymorphisms.

\section{Acknowledgements}

We sincerely thank Dr Jacques L. David and Professor André Charrier for critical reading of the manuscript; and MM. Guela Bledy Felix, Boka Clément and Bessi Toba Gérard for their technical help. This paper was written during a stay at the Station de génétique végétale at Gif-sur-Yvette.

This work was supported in part by a grant from the Commission of European Communities (TSD A $103 \mathrm{~F})$.

\section{References}

ATTERE, A. F. AND FATOKUn, C. A. 1983. Reaction of O. glaberrima accessions to rice yellow mottle virus. Plant Disease, 67, 420-421.

BOUGEROL, B. AND PHAM, J. L. 1989. Influence of the Oryza sativa genotype on the fertility and quantitative traits of $\mathrm{F} 1$ hybrids between the two cultivated rice species $O$. sativa L. and $O$. glaberrima Steud. Genome, 32, 810-815.

CAusse, M., AND GHEsouiere, A. 1991. Prospective use of $O$. longistaminata for rice breeding. In: IRRI (ed.) Rice genetics II, Proceedings of the Second Rice Genetics Symposium, 14-18 May 1990, Manila, Philippines pp. 81-89.
CHU, Y. E., MORISHMAN, H. AND OKA, H. 1. 1969. Reproductive barriers distributed in cultivated rice species and their wild relatives. Jpn. J. Genet., 44, 207-233.

CHU, Y. E. AND OKA, H. I. 1970. The genetic basis of crossing barriers between Oryza perennis subsp. barthii and its related taxa. Evolution, 24, 135-144.

DALly, A. AND SECOND, G. 1990. Chloroplast DNA diversity in wild and cultivated species of rice (Genus Oryza, section Oryza). Cladistic mutation and genetic-distance analysis. Theor. Appl. Genet., 80, 209-222.

GADISH, I. AND ZAMIR, D. 1987. Differential zygotic abortion in an interspecific Lycopersicon cross. Genome, 29, $156-159$.

GHESQUiÈRE, A. AND SECOND, G. 1983. Polymorphisme enzymatique et évolution d'O. sativa en Afrique. In: Colloque Electrophorèse et Taxonomie. Société Zoologique de France. pp. 263-272.

GHesquiere, A. 1988. Diversité de l'espèce sauvage de riz Oryza longistaminata $A$. Chev. \& Roehr et dynamique des flux géniques au sein du groupe Sativa. Thèse Doctorat es Science, Univ. Paris Sud Orsay, pp. 228.

GRANT, v. 1975. Genetics of Flowering Plants. Columbia University Press, New York and London.

IKEHASHI, H. AND ARAKI, H. 1986. Genetics of remote crosses of rice. In: IRRI (ed.) Rice Genetics, Proceedings of the First Rice Genetics Symposium, IRRI, Manila, pp. 119-130.

IKEHASHI, H., YANAGIHARA, S. AND HARAKI, H. 1991. Screening and analysis of wide compatibility loci in wide crosses of rice. In: IRRI (ed.) Rice Genetics II. Proceedings of the Second Rice Genetics Symposium, IRRI, Manila, pp. 33-43.

JOHN, V. T. AND THOTTAPILLY, G. 1987. A scoring system for rice yellow mottle virus disease (RYMV). Int. Rice. Res. Newsl., 12, 26.

JOHN, v. T., THOTTAPILLY, G., NG, Q., ALURI. K. AND GIBBONS, J. N. 1985. Varietal reaction to rice yellow mottle virus disease. FAO Plant Protection Bull., 33, 109-111.

KOCHKO, A. DE 1987. Isozymic variability of traditional rice (Oryza sativa L.) in Africa. Theor. Appl. Genet., 73, 675-682.

MORINAGA, T. 1964. Cytogenetical investigations on Oryza species. In: Rice Genetics and Cytogenetics. Proceedings of the Symposium on Rice Genetics and Cytogenetics. Los Banos, Philippines, February 4-8, 1963. Elsevier, Amsterdam, pp. 91-103.

MORISHIMA, H., HINATA, K. AND OKA, H. I. 1962. Comparison between two cultivated rice species Oryza sativa L. and $O$. glaberrima Steud. Jpn. J. Breed. 12, 153-165.

OKA, H. I. 1988. Origin of Cultivated Rice. Developments in crop science, 14. Japan Scientific Societies Press, Tokyo.

otTaviano, E. AND mulCAHY, D. L. 1986. Gametophytic selection as a factor of crop plant evolution. In: Barrigozi, C. (ed.) The Origin and Domestication of Cultivated Plants. Symposium, 25-27 November 1985, Rome, Italy, Amsterdam, Netherlands, Elsevier pp. 101-120.

PATERSON, A. H. DE VERNA, J. W., LANINI, B. AND TANKSLEY, S. D. 1990. Fine mapping of quantitative trait loci using selected overlapping recombinant chromosomes in an interspecies cross of tomato. Genetics, 124, 735-742. 
PHAM, J. L., GLASZMANN, J. C., SANO, R., BARBIER, P., GHESQUIËRE, A., AND SECOND, G. 1990. Isozyme markers in rice: genetic analysis and linkage relationships. Genome, 33, 348-359.

RICK, C. M. 1963. Differential zygotic lethality in tomato species. Genetics, 48, 1497-1507.

SANO, Y. 1983. A new gene controlling sterility in $F_{1}$ hybrids of two cultivated rice species. Its association with photoperiod sensitivity. J. Hered., 74, 435-439.

SANO, Y. 1986. Sterility barriers between Oryza sativa and $O$. glaberrima In: IRRI (ed.) Rice Genetics. Proceedings of the First Rice Genetics Symposium, IRRI, Manila, pp. 91-107.

SANO, Y. 1990. The genic nature of gamete eliminator in rice. Genetics, 125, 183-191.

SANO, Y., CHU, Y. E. AND OKA, H. I. 1979. Genetic studies of speciation in cultivated rice. 1. Genic analysis for F1 sterility between $O$. sativa L. and $O$. glaberrima Steud. Jpn. J. Genet, 54, 121-132.

SANO, Y., CHU, Y. E. AND OKA, H. 1. 1980. Genic studies of speciation in cultivated rice. 2. Character variations in backcross derivatives between $O$. sativa and $O$. glaberrima: $\mathrm{M}-\mathrm{V}$ linkage and key characters. Jpn. J. Genet., 55, 19-39.

SANO, Y., SANO, R. AND MORISHIMA, H. 1984. Neighbour effects between two occurring rice species, Oryza sativa and $O$. glaberrima. J. Appl. Ecol., 21, 245-254.

SAUPHANOR, B. 1985 . Some factors of upland rice tolerance to stemborers in West-Africa. Insect Science and its Application, 6, 429-434.

SECOND, G. 1982. Origin of the genetic diversity of cultivated rice (Oryza spp): study of the polymorphism scored at 40 isozyme loci. Jap. J. Genet., 57, 25-57.

SiLUE, D. AND NOTTEGHEM, J. L. 1991. Resistance of 99 Oryza glaberrima varieties to blast. Int. Rice Res. Newsl., 16, 13-14.

SOKAL, R. R. AND ROHLF, F. J. 1981. Biometry. The Principles and Practice of Statistics in Biological Research. Freeman and Co, San Francisco.

TROUSLOT, P. AND SECOND, G. 1980. Technique d'électrophorèse en gel d'amidon appliquée à l'étude du polymorphisme de 14 enzymes de riz. In: Electrophorèse d'enzymes de riz. Paris, ORSTOM (Travaux et Documents de l'ORSTOM $\left.n^{\circ} 120\right)$.

Vercambre, B. 1982. Diopsis thoracica West (Dipt. Diopsidae), important ravageur du riz en Afrique de l'Ouest: données bio-écologiques et application à la lutte intégrée. Agron. Trop., 37, 89-98.

WANG, Z. Y., SECOND, G. AND TANKSLEY, S. D. 1992. Polymorphism and phylogenetic relationships among species in the genus Oryza as determined by analysis of nuclear RFLPs. Theor. Appl. Genet., 83, 565-581.

WENDEL, J. F., EDWARDS, M. D. AND STUBER, C. w. 1987. Evidence for multilocus genetic control of preferential fertilization in maize. Heredity, 58, 297-301.

YABUNO, T. 1977. Genetic studies on the interspecific cytoplasm substitution lines of japonica varieties of $O$. sativa L. and $O$. glaberrima Steud. Euphytica, 26, 451-463.

yabUnO, T. 1990. A gametocidal factor of Oryza glaberrima Steud in O. sativa L. Euphytica, 45, 191-195. 